\title{
Methodology for the integrative adaption of manufacturing process and inspection sequences to component changes of safety-critical medical products
}

\author{
Lars Stauder $^{1}$ [D Anna-Lena Knott ${ }^{2} \cdot$ Robert H. Schmitt $^{2,3} \cdot$ Thomas Bergs $^{1,3}$
}

Received: 7 December 2021 / Accepted: 2 February 2022 / Published online: 17 February 2022

(c) The Author(s) 2022

\begin{abstract}
Ever-shorter product lifecycles and more frequently changing customer demands challenge manufacturing companies to change their products and the components these products are composed of in ever-shorter periods. As a result, the existing manufacturing process and inspection sequences (MPISs) of the corresponding components must be adapted. Particularly in the production of safety-critical components, such as in the medical industries, component changes represent a major challenge, as very high quality requirements are placed on the components and a costly re-certification of the adapted processes is necessary. Due to the high quality requirements, not only the manufacturing process but also the inspection processes must be adapted for the realization of a component change. Furthermore, a high degree of planning reliability is necessary when deriving adaptations of MPISs for component changes to keep the re-certification effort and adaptation costs for safety-critical components as low as possible. Therefore, a methodology is introduced for the integrative derivation of adaptation options of MPISs due to component changes that supports users in identifying suitable adaptations in a systematic and time-efficient way. The methodology is successfully applied to a use case from the medical industry and supports users in identifying adaptations in established MPISs to implement a component change.
\end{abstract}

Keywords Technology planning $\cdot$ Manufacturing change management $\cdot$ Medical industry $\cdot$ Inspection adaptation $\cdot$ Safetycritical

\section{Introduction}

Ever faster-changing customer needs and the increasing individualization of products, as well as shorter product life-cycles result in more frequent adaptations of products and therefore, the underlying production processes [1,2].

Lars Stauder

L.Stauder@wzl.rwth-aachen.de

https://www.wzl.rwth-aachen.de

1 Chair for Manufacturing Technologies of the Laboratory for Machine Tools and Production Engineering (WZL), RWTH Aachen University, Campus-Boulevard 30, 52074 Aachen, Germany

2 Chair for Production Metrology and Quality Management of the Laboratory for Machine Tools and Production Engineering (WZL), RWTH Aachen University, Campus-Boulevard 30, 52074 Aachen, Germany

3 Fraunhofer Institute for Production Technology IPT, Steinbachstr. 17, 52074 Aachen, Germany
Manufacturing companies are increasingly faced with the challenge of adapting their production to product changes, such as the modification of a component characteristic [3, 4].

Manufacturing process and inspection sequences (MPISs) comprise the combined consideration of sequential manufacturing and inspection processes and are used to display or model the production process of components [5]. Adaptations to implement production changes include both changes to manufacturing and inspection processes. A change in production can be either a technological change (e.g. change of lapping parameters due to a change in the surface) or a scheduling change (e.g. a change in batch size). Scheduling changes usually do not have a direct influence on the design of the MPIS. Therefore, this paper focuses on technological changes in the form of changes of a component characteristic and resulting manufacturing and inspection changes.

Particularly for the production of safety-critical components, such as in medical technology, component changes pose a major challenge as re-certification is necessary to ensure high quality requirements [6-8]. The profitability of 
adaptation options for implementing component changes is additionally highly influenced by re-certification costs. Also, the duration of the change implementation is an important factor. Therefore, a high degree of planning reliability is required when deriving adaptations to keep the re-certification effort and adaptation costs for component changes as low as possible [6].

Due to strict legal requirements to ensure the functionality of safety-critical components, the contribution of quality costs to the overall production costs can even exceed the manufacturing costs [9]. Therefore, it is not sufficient to solely consider the adaptation of manufacturing processes when implementing a production change. [10]. The effects of the manufacturing change on the inspection sequence have to be considered, as the scrap rate or the suitability of measurement equipment might change as a result of the manufacturing change.

Therefore, this paper presents a methodology for the integrative derivation of adaptation options for MPISs due to component changes in a systematic and time-efficient way.

In the following, the state of research on the identification of manufacturing and inspection changes is analyzed and the research gap is identified. Subsequently, the methodology for the integrative derivation of adaptations for MPISs due to component changes is presented and validated by application to a use case from medical technology. Finally, a summary of the research results and an outlook on further research are given.

\section{State of research regarding the integrative identification of manufacturing and inspection changes}

The analysis of the state of the art focuses particularly on the research field of manufacturing change management (MCM). Furthermore, approaches from inspection and technology planning are examined, since these fields deal with the identification of suitable manufacturing or inspection processes and the generation and design of process and inspection sequences [11]. MCM derives from Engineering Change Management (ECM) [12]. While ECM focuses on product changes, MCM deals with process changes [12]. An overview of relevant approaches from MCM for the planning of manufacturing changes is given by Hermann et al. [6]. In the following, the most relevant approaches for this research are presented.

Marks et al. developed a systematic approach for modeling the capabilities of manufacturing processes and the dependencies between a manufacturing process and the produced component, which enables the derivation of possible manufacturing changes to implement a component change [13]. However, the approach only addresses individual processes and not process sequences. Inspection processes are not taken into account either.

Karl et al. developed a methodology to identify and plan reconfigurations for manufacturing resources such as machine tools or assembly systems to support cost-efficient adaptations to changed circumstances [14]. Component changes are not explicitly considered. Hoang et al. presented a systematic approach to generate adaptation options for manufacturing machines considering constraints and interrelationships [15]. Due to the focus on single manufacturing machines, MPISs are not considered.

Masood et al. presented an approach for modeling value chains and interdependencies between manufacturing processes and components to identify factors influencing the changeability or flexibility across value chains [16]. This supports users in identifying levers for implementing manufacturing changes. Yet, the approach neglects changes in the inspection sequence.

Cichos et al. introduced a framework for the physical modeling of an established manufacturing process sequence to support the implementation of component changes [17]. However, the identification and derivation of adaptations of the MPIS are not part of the approach.

Plehn developed a method for analyzing the impact of changes and their propagation in manufacturing systems, whereby the reference frame comprises a complete factory and the identification of specific manufacturing changes on process level is not considered [18].

Thus, the approaches from Masood et al., Cichos et al. and Plehn can support users in identifying meaningful levers for implementing manufacturing changes, for example, because of the change of a supplier or a change in the company's policy. Component changes as triggers of a manufacturing change are not addressed.

Hermann et al. presented a systematic method for generating manufacturing changes for safety-critical components due to a component change. In addition, the method supports the user in the analysis of the influence of manufacturing changes on the entire manufacturing process sequence [6]. However, inspection processes are not part of the approach.

Müller developed a methodology for the integrative technology and inspection planning of medical devices. The aim is to support technology planners in designing economically optimized MPISs. Thus, the approach considers both manufacturing processes and inspection [19]. However, the focus is on the initial design of the MPIS for a new product. Component changes of established products are not within the scope of the approach and the design of new MPIS conflicts with a time- and cost-efficient implementation of the component change and possible re-certification, since a complete replanning for the implementation of a change is associated with large expenses. Klocke et al. introduced a similar 


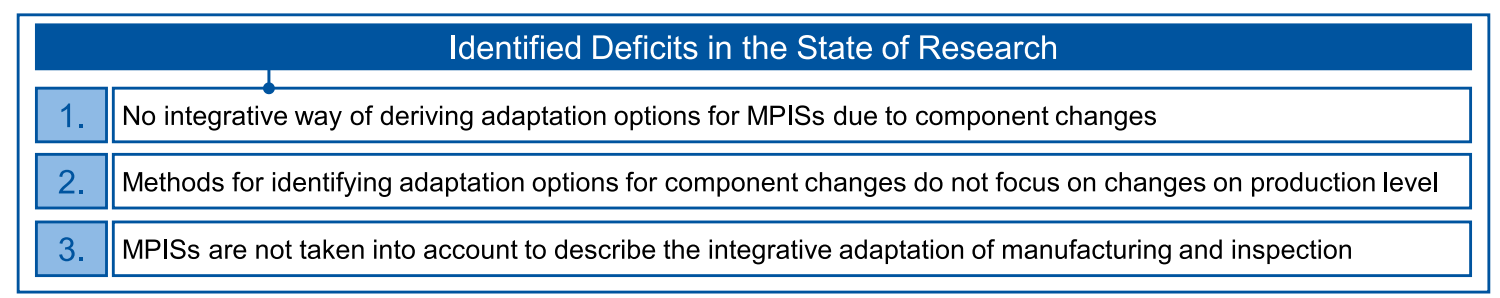

Fig. 1 Deficits of the state of research

approach to Müller with identical deficits in terms of the identification of manufacturing and inspection changes [5].

Schmidt introduced a methodology for integrative planning and production control based on condition information to increase the flexibility of manufacturing to react to disruptions [20]. Due to the high flexibility of the manufacturing chain, which is achieved with the approach, a quick reaction to changed production needs is possible. Component changes and inspection processes are not discussed explicitly within the approach. Other approaches that base on an established manufacturing process sequence are, for example, the approaches of Denkena et al. for the production planning in highly dynamic environments [21] or Beckers et al. for the design of economically-optimized manufacturing process sequences [22]. Similar to Schmidt's approach, these approaches offer methods to implement a component change. However, those methods are not systematically combined and there are no guidelines for the efficient derivation and implementation of component changes. Furthermore, inspection processes are not part of the approaches.

Inspection planning particularly addresses aspects of the derivation of adaptations for inspection sequences due to a component change. Basse et al. address the evaluation of alternative inspection strategies according to the resulting costs. [23] How the alternatives are generated is not focussed.

The stage-gate model developed by Wuest et al. presents a possibility to describe the product status along an MPIS. [24] It enables the derivation of the initial inspection sequence to a given manufacturing chain. However, the approach neglects component changes or the generation of MPISs.

The analysis of the state of research, summarized in Fig. 1, shows that none of the existing approaches enables an integrative derivation of adaptations to MPISs due to component changes. Some of the presented approaches provide useful methods and models for identifying adaptations for the implementation of component changes. However, the focus is usually not on changes in production. In addition, with exception of Müller's approach, none of the approaches takes MPISs into account, which is why they cannot be used for an integrative consideration of manufacturing and inspection processes. Consequently, they cannot be used to solve the research problem appropriately and further research is needed. Nevertheless, they serve as a basis for solving the research problem. A methodology is presented below that supports technology planners in the systematic identification of adaptation options for implementing a component change in MPISs for safety-critical components.

\section{Methodology and application for the integrative derivation of adaption options for the implementation of component changes}

The methodology's basis is an already implemented MPIS and a known component change. The methodology aims to systematically identify suitable adaptation options to implement the component change in this MPIS and is divided into three steps shown in Fig. 2. Firstly, all relevant manufacturing process steps are identified, which influence the component characteristic under consideration. Secondly, suitable manufacturing adaptation options to implement the component change are derived. Thirdly, for each possible manufacturing adaptation option, the existing inspection sequence is analyzed for necessary changes due to the manufacturing adaptation to ensure the high product quality standards. The inspection strategy is based on the manufacturing process sequence and is therefore analyzed sequentially to the second step of deriving manufacturing changes. For identifying the most economical and time-efficient change in manufacturing and inspection an evaluation considering amongst others the implementation and possible re-certification effort needs to be carried out. This requires a separate method, the description, and execution of which is beyond the scope of this publication. Therefore, the methodology presented focuses solely on the creation of adaptation options for MPISs due to component changes.

The use case, to which the method for deriving adaptation options for MPISs due to component changes is applied, is the production of the lower body of a left ventricular assist device (LVAD), which supports the heart by continuously accelerating blood through a hydrodynamically supported 


\section{를 - Existing MPIS \\ - Required component change}

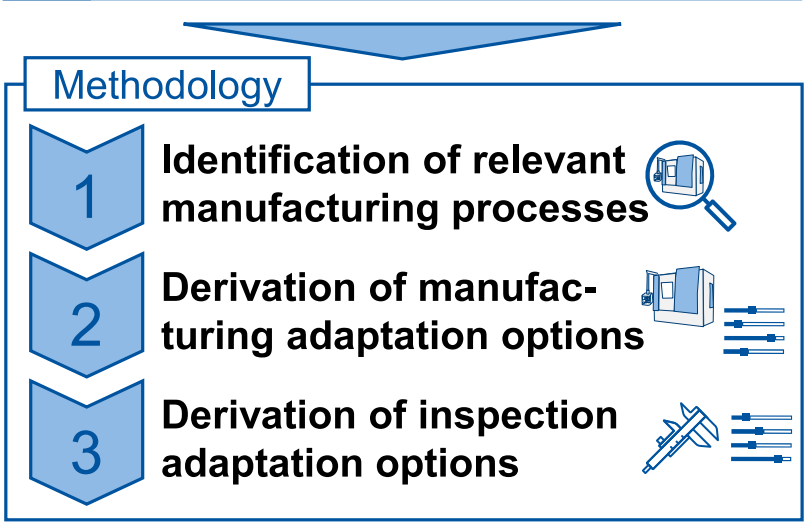

$+2$

\section{흥 - Possible adaptation options for 을 implementing component change}

Fig. 2 Overview of the methodology

rotor. The lower body of the LVAD is manufactured via different manufacturing processes, namely milling, drilling, lapping, and drag grinding. Additionally, inspection processes are conducted to ensure the quality of the component.
The MPIS of the use case is depicted in Fig. 3. As a component change, a change in the surface roughness of the blood channel from 0.1 to $0.08 \mu \mathrm{m}$ is considered.

\subsection{Identification of relevant manufacturing processes}

To analyze, how the component change affects the MPIS, all relevant manufacturing processes are identified that directly influence the final state of the considered component characteristic, the surface roughness of the blood channel. To support the user in identifying these processes within the manufacturing process sequence, Müller's approach for modeling dependencies within manufacturing process sequences is used (cf. [19]) and extended by a matrix. An extract of the modeling of the manufacturing process sequence focused on the surface roughness of the blood channel of the lower body of the LVAD is shown in Fig. 4 and is explained in the following.

Within the model, the lines illustrate whether a manufacturing process influences a characteristic (dashed lines). The symbols indicate whether a manufacturing process newly generates characteristics (rhombus) or modifies them (circle). Not every process that influences a component characteristic influences the final state of the component characteristic. For example, a milling process creates a new micro-geometry. Therefore, the surface roughness before the milling process and thus the processes which

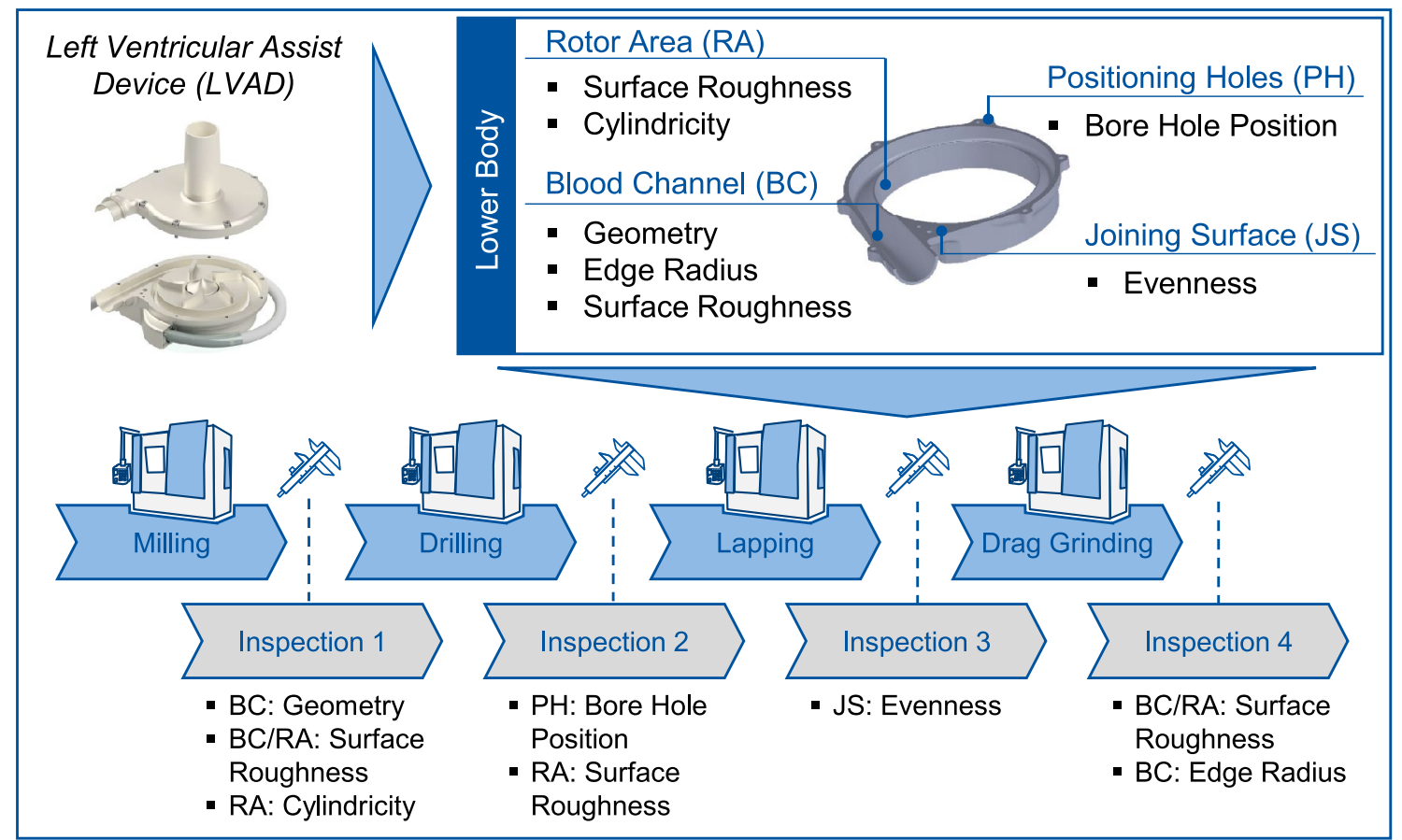

Fig. 3 Introduction of the use case 


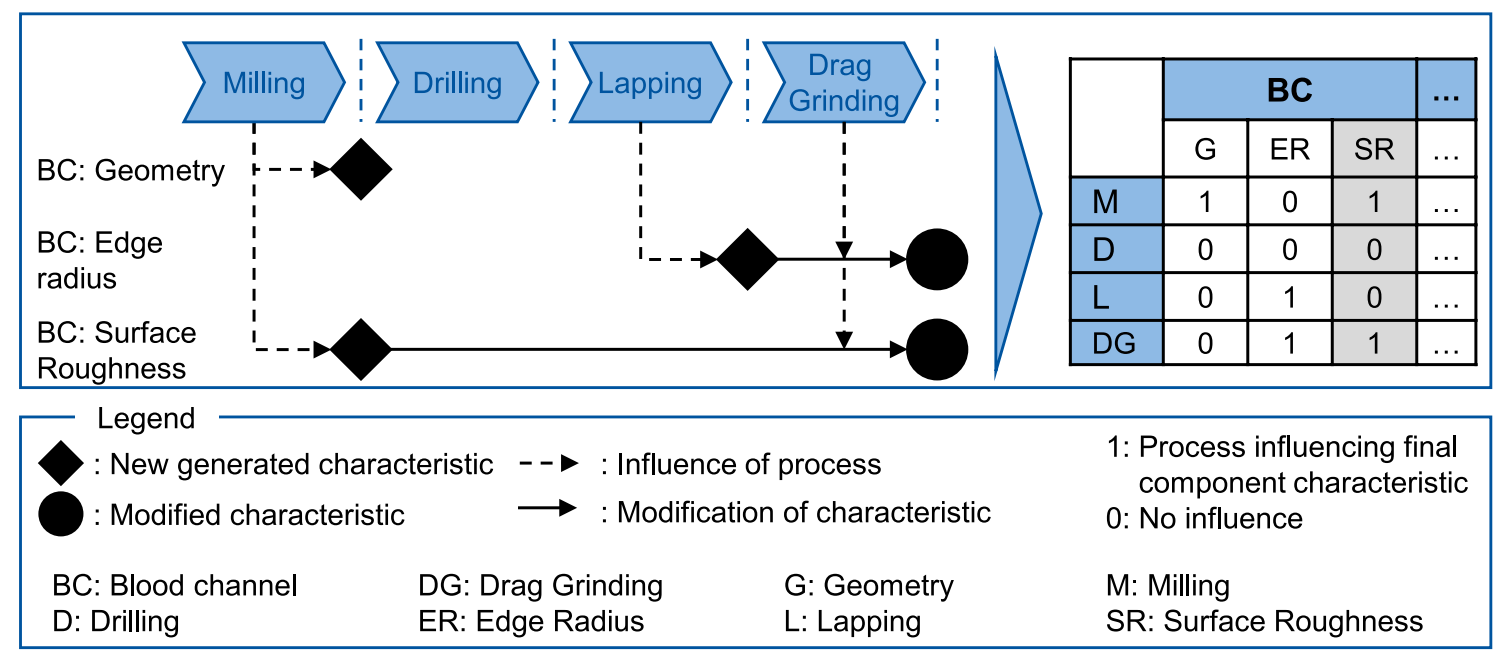

Fig. 4 Modelling of MPIS of the use case and identification of relevant manufacturing processes

influence this previous surface roughness, do not influence the final surface roughness of the component. In addition to Müller's approach, the results of the modeling of the dependencies within manufacturing process sequences are summarised in a matrix (see Fig. 4 on the right). In the rows of the matrix the different manufacturing processes and in the columns the component characteristics are listed. Within the matrix, a " 1 " represents an influence of the manufacturing process on the component characteristic. To determine which processes influence which component characteristics, comprehensive technology knowledge and support from technology experts is necessary. Levers for implementing the component change can be applied to the processes that influence the final state of the component characteristic. Exemplary for the use case, the blood channel is described by three component characteristics (geometry, edge radius, and surface roughness). Milling and drag grinding have an influence on the final surface roughness of the blood channel and therefore adaptations of these processes are possibilities to implement the change in the surface roughness.

\subsection{Derivation of manufacturing adaptation options}

After identifying the processes that influence the final component characteristic, the next step is to derive adaptation options to implement the component characteristic change. The classification model for adaptation options shown in Fig. 5 is used to identify adaptation options time- and costefficiently. The model is based on the model from Bergs et al. for basic adaptations in manufacturing (cf. [2]) and was extended and adapted for the method.

The adaptation options are categorized into three classes based on their scope and level of detail. The first class, process, describes software adaptations on the machine control level and comprises adaptations of the process parameters and the operating procedure as well as aggregations or separations of process steps (e.g. roughing and finishing during milling). The second class, machine, includes hardware changes of the machine such as effect pair change (tool/ component), supply change as well as machine adaptation or replacement. The third class, structure, describes adaptations of the manufacturing process sequence such as sequence change, process substitution, or process sequence extension or contraction.
Fig. 5 Classification model of adaptation options

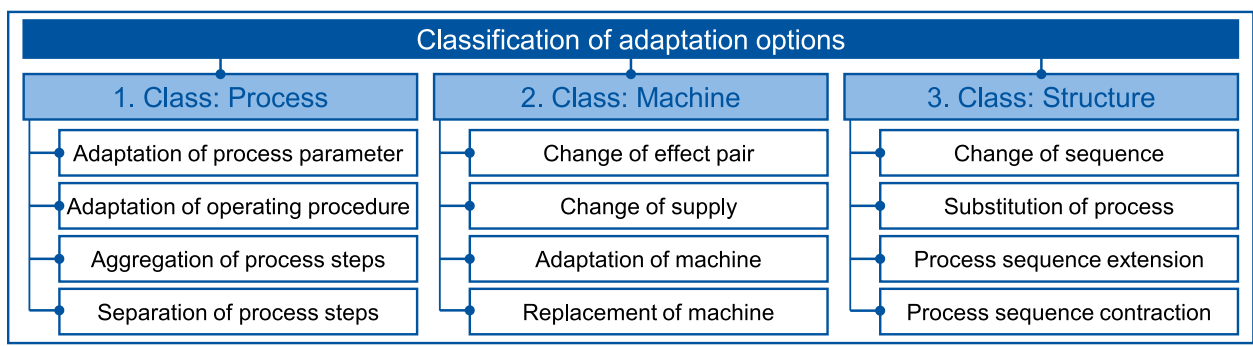


Based on the previously identified manufacturing processes which influence the surface roughness of the lower body of the LVAD, it is successively checked whether suitable adaptation options for implementing the change exist within a class. The classes are examined by increasing scope (process $\rightarrow$ machine $\rightarrow$ structure). This guarantees that less time-consuming adaptation options with little certification, planning and implementation effort are evaluated for their technical feasibility first. For example, changing the supply or the machine is more cumbersome then the adaptation of a process parameter. Furthermore, the classes build on each other, so the implementation of measures of higher classes generally also require measures of lower classes. Within a class, there is no indication for options with the lowest costs and effort, therefore, various alternative options are to be examined. For each category of the class (e.g. process parameter change) factors that influence the considered component characteristic (e.g. surface roughness) are identified. For example, not every process parameter in milling influences the surface roughness. In addition to empirical knowledge technological models (e.g. [25] or [26]) help identifying relevant factors.

Following the identification of the relevant factors, the adaptation options of the factors are analyzed for the suitability of realizing the required component change. Due to the special requirements for the manufacturing of safety-critical components and the company-specific manufacturing, extensive and detailed process knowledge from e.g. technology experts must be used. The result of this step are potential adaptation options for implementing the required change. For the holistic consideration of manufacturing and inspection, the most appropriate adaption option can only be identified after examining the inspection sequence.

For the use case, two processes (milling and drag grinding) with influence on the final surface roughness of the blood channel were identified. The two processes are investigated separately regarding potential adaptation options, starting with options from the process level. Since the final surface roughness is directly influenced by the process time of the drag finishing process, the reduction in surface roughness can be achieved by increasing the process time $\left(A_{1}\right)$. The surface roughness can also be reduced by adapting the operating procedure of the drag grinding by adding a finishing cycle $\left(\mathrm{A}_{2}\right)$. Since possible adaptation options for the drag grinding process could already be found in the process classes, the other classes are not further investigated.

Through an adaptation of the milling process parameters, the resulting surface roughness can be reduced, e.g. reducing the feed rate. However, in this use case, the feed rate cannot be reduced enough to achieve the required surface roughness. Other adaptations at the process level are not suitable either. The analysis is therefore extended to the machine level. Changing the milling tool (effect pair change) to a suitable tool for achieving a lower surface roughness is a possible adaptation option $\left(\mathrm{A}_{3}\right)$. To exploit the dependencies of processes a combination of adaptation options is also possible. For example, the surface roughness after drag grinding is directly dependent on the surface roughness after milling. The lower the roughness after milling, the lower the roughness after drag grinding will be (if all other conditions are equal). A combined adaptation (reduction of the feed rate for milling and increase of the process time for drag grinding) can also fulfill the requirement of the component change $\left(\mathrm{A}_{4}\right)$.

In some cases, the extension of the scope to the third class is usefull, even if adaptation possibilities have already been identified (e.g. if free capacities on additional machines are available, or the other adaptation options are explored and do not lead to the desired results). A possible way to fulfill the requirements for the lower surface roughness is to extend the process sequence with an additional finishing process, e.g. polishing $\left(\mathrm{A}_{5}\right)$.

Five exemplary adaptation options $\left(\mathrm{A}_{1}-\mathrm{A}_{5}\right)$ to implement the component characteristic change were identified (see Fig. 6). It is necessary to analyze whether unintended effects, e.g. on component characteristics that are not supposed to be changed, are associated with the implementation of the adaptation options. These unintended effects result from the complex dependencies within a manufacturing process sequence. Therefore, additional measures may be necessary for the successful implementation of an adaptation option. A method for the identification of unintended effects

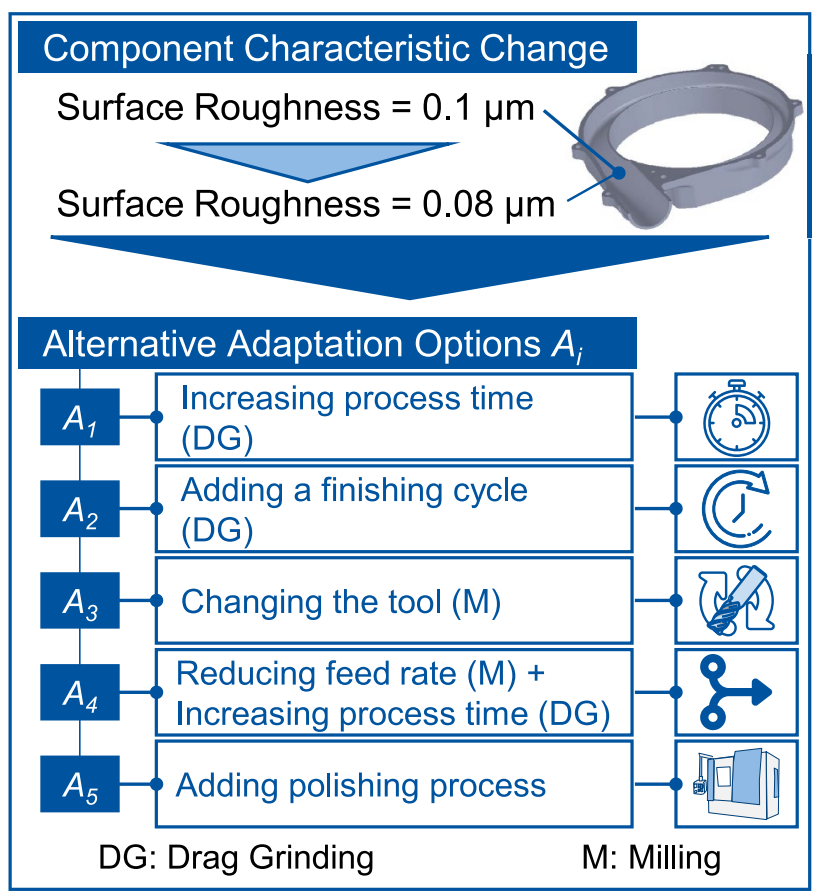

Fig. 6 Alternative adaptation options of the use case 
is presented by Bergs et al. [4] and will not be addressed in this paper.

\subsection{Derivation of inspection adaptation options considering manufacturing changes}

The inspection type and extent of features are highly dependent on the manufacturing steps that led to the creation of the component's features. Therefore, the next step, analyzing whether any adaptations of the manufacturing process sequence lead to necessary adaptions of the inspection sequence, is subsequent to the derivation of manufacturing changes. Adaptations of the inspection sequence are analyzed for every possible manufacturing change in Sect. 3.2. The economical evaluation between options requires an overview over all possible MPIS reacting to the component change.

$A_{1}-A_{4}$ focus on the adaptation of an already implemented process. For the use case, it is assumed that the changed process parameters do not influence the scrap rate of the process negatively. Therefore, only $\mathrm{A}_{5}$ leads to an adaptation of the inspection sequence. For a detailed description of possible cases during the adaptation of an inspection sequence, the adaptation option $\mathrm{A}_{5}$ is analyzed in the following paragraphs.

For being able to derive inspection adaptation options from the manufacturing changes, the initial creation of the inspection sequence needs to be understood. Müller developed a set of rules to establish which characteristic needs to be inspected in which checkpoint and to which extent. Checkpoints are located between two successive manufacturing processes and represent potential moments to inspect one or more component characteristics (see dashed lines between the manufacturing processes in Fig. 4). [19]

Müller then describes three possible types of inspection [19]. A mandatory inspection of a component characteristic is required to ensure the final quality of the component when the preceding process is the final manufacturing process changing the characteristic. The mandatory inspection in the checkpoint after the last influence on the feature through a manufacturing process corresponds to a step-wise end-of-line inspection and ensures, that every features last state is inspected. The either/or inspection is used for characteristics, that are not changed along the rest of the process sequence. The characteristic needs to be inspected at least once during the rest of the process sequence and preferably at the first possible inspection point to identify scrap as early in the process sequence as possible. This is based on the assumption that the inspection costs do not differ depending on the position of the inspection in the process sequence. If this assumption is not true, an either/or inspection in the latter stages need to be considered. Optional inspections are set in every checkpoint for every characteristic that influences other characteristics in a process. This supports the identification of re-work and scrap as early in the process sequence as possible. If a characteristic is not changed during the process sequence, but it influences other characteristics a combination of optional and either/or inspections is inserted in the process sequence as both the condition for the either/or and the optional inspection are applicable. Figure 7 shows the result for the inspection sequence in the use case following the rules by Müller. As characteristic M1.1 is only influenced by the first manufacturing process an either/or inspection is chosen. It has to be inspected at least once during the process sequence.
Fig. 7 Graph for deriving the initial inspection strategy for the blood channel

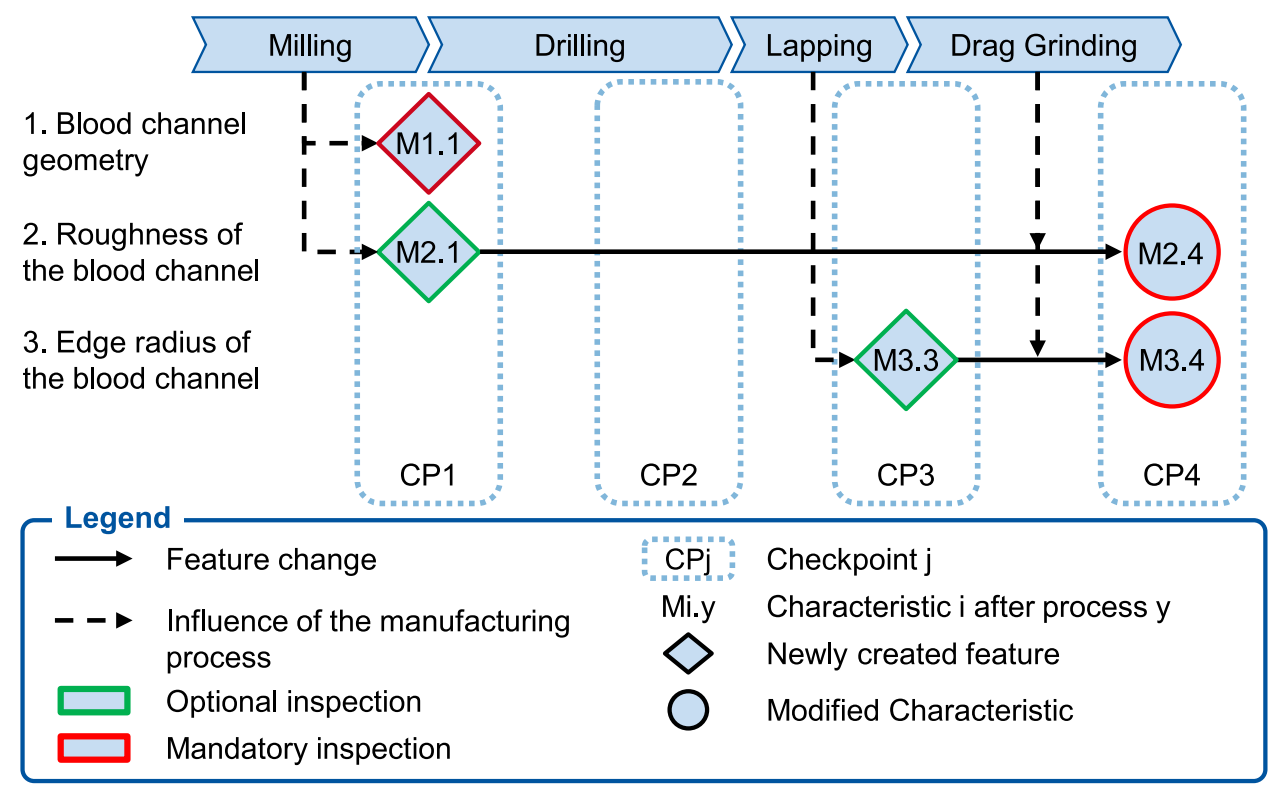


The earliest possible inspection point is the most economical as this way scrap can be identified earliest in the process and is therefore chosen for inspection of characteristic M1.1. The optional inspections lead to different possible inspection sequences. The most cost-effective was selected after an economic evaluation.

Figure 8 shows the resulting inspection strategy for the blood channel that is chosen for the use case as a result of the evaluation of possible strategies. The manufacturing adaptations identified in Sect. 3.2 are now analyzed on their effect on the initial inspection strategy to assess whether and which adaptations of the inspection sequence are necessary.

The different measures to implement the component change in manufacturing can be divided into two groups regarding their influence on the inspection sequence. Firstly, the addition of a process step, or secondly the adaptation of an existing process step.

To derive inspection adaptations from the manufacturing change four possible adaptations for an existing inspection process are possible. The relocation of the inspection process in the process sequence to a different checkpoint, the deletion of an inspection process that became obsolete, or the addition of an inspection process, if the scrap rate of a process increased significantly. The fourth option is not a change in the placement of the inspection but a change in the measuring equipment, because the previously used equipment is no longer capable of inspecting the characteristic, for example, because the measurement uncertainty is too high compared to the tolerance of the characteristic.

If a new manufacturing process is added, the newly created checkpoint needs to be integrated and evaluated according to the initial creation of the inspection sequence following the rules by Müller [19]. With adaptation option $\mathrm{A}_{5}$ a new last process and therefore an inspection 5 is integrated into the manufacturing sequence, see Fig. 9. As for safety-critical components, all characteristics must be inspected after the last manufacturing process that influences them. Therefore, every characteristic that is influenced by the new manufacturing process needs to be shifted from inspection 4 to inspection 5 . In this case, the drag grinding process is not expected to produce a high scrap rate that would indicate additional inspections in checkpoint 4. Figure 9 shows the example of the derived inspection sequence after modification $\mathrm{A}_{5}$ is implemented.

If the added process is not at the end of the process sequence, an analysis of the dependencies within the manufacturing process sequence is required to identify if an additional inspection is required. Müller provides a method to analyze the interdependencies for the initial creation of the MPIS, see Fig. 4 [19]. Mandatory inspections need to be set, where influences from the newly added process on unchanged characteristics are identified. In the use case, the

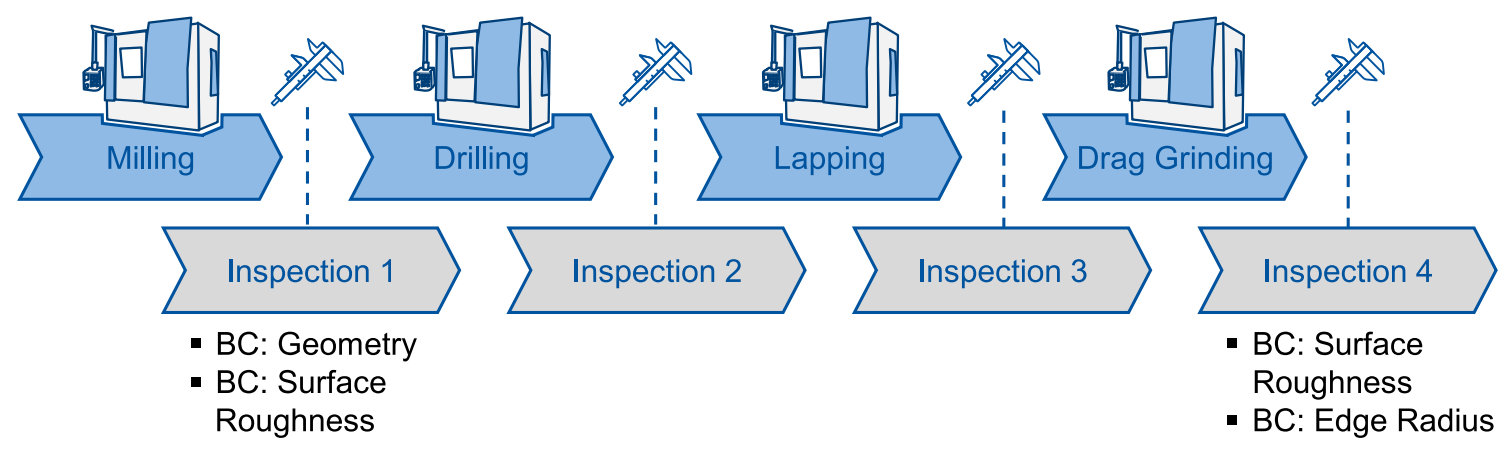

Fig. 8 Initial MPIS for the blood channel (BC) from the use case

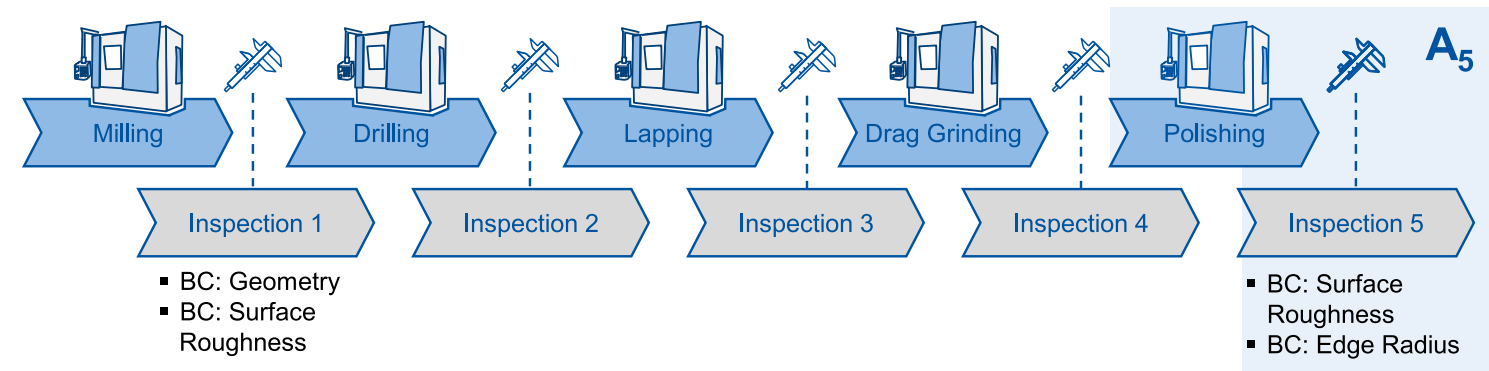

Fig. 9 Adjusted inspection sequence for adaptation option $\mathrm{A}_{5}$ 
added process is a new last process in which every characteristic considering the blood channel is inspected because of a direct influence of the new process.

If the manufacturing change is only adapting process parameters the manufacturing principle is not changed and therefore the interdependencies between processes and characteristics are not changed. To analyze whether there is a need for a change of the inspection strategy the scrap rate of the adapted process needs to be analyzed. If a process change leads to a higher scrap rate, the setting of an obligatory inspection process after the process needs to be evaluated. Only if the scrap rate becomes significantly higher, the inspection becomes necessary. Otherwise, the former inspection strategy is kept. The limit value of the scrap rate is individually set by process experts. Accordingly, at least one optional inspection at the earliest possible inspection point needs to be placed during the rest of the process sequence. The alternative adaptations $\mathrm{A}_{1}-\mathrm{A}_{4}$ for the use case only address an adaptation of the manufacturing processes. An increased scrap rate is not expected. Therefore, these options do not make the change of the inspection strategy necessary.

With the methodology presented in this paper, a component change of a safety-critical product from medical technology is successfully implemented, firstly in the adaptation of the existing manufacturing process chain. Afterward, the inspection sequence is adapted according to the changes in manufacturing to implement the component change in the MPIS.

\section{Summary and outlook}

A methodology for the integrative derivation of adaptation options of MPISs due to component changes was presented. It is divided into three steps. Firstly, a procedure for the identification of relevant manufacturing processes influencing the considered component characteristic is introduced. Secondly, a classification model for supporting users in deriving suitable manufacturing adaptation options to implement the component change is developed. Thirdly, the existing inspection sequence is analyzed to determine whether the change in the manufacturing processes necessitates adaptations to ensure the high demands on product quality. Adaptations are analyzed sequentially to the second step of the evaluation of manufacturing changes because the inspection strategy is based on the manufacturing process sequence.

The methodology was then successfully applied to the production of the lower body of an LVAD, as a use case from the medical industry. It is shown that the methodology supports users in identifying adaptations in established MPISs to implement a component change. To further enhance the industrial applicability of the presented methodology, additional use case studies should be conducted.

Further research is needed to evaluate the possible adaptation options to determine the technologically and economically optimized option. The systematic evaluation method of the adaption options should only consider those cost factors that are influenced by the adaptation option so that a timeand cost-efficient evaluation is possible. Furthermore the evaluation focuses on a time and cost-efficient realization of re-certification efforts with the adaption options. In addition, the inspection relevance of the component characteristics is to be integrated into the consideration, as this enables a more well-founded derivation of necessary adaptations of the inspection strategy.

Acknowledgements The authors would like to thank the Deutsche Forschungsgemeinschaft (DFG, German Research Foundation) for funding this research within the project "Kostenoptimierte medizintechnische Herstellprozesse durch Integration von Technologie- und Prüfplanung (IMHOTEP)" Project-ID: 323577145.

Funding Open Access funding enabled and organized by Projekt DEAL.

Open Access This article is licensed under a Creative Commons Attribution 4.0 International License, which permits use, sharing, adaptation, distribution and reproduction in any medium or format, as long as you give appropriate credit to the original author(s) and the source, provide a link to the Creative Commons licence, and indicate if changes were made. The images or other third party material in this article are included in the article's Creative Commons licence, unless indicated otherwise in a credit line to the material. If material is not included in the article's Creative Commons licence and your intended use is not permitted by statutory regulation or exceeds the permitted use, you will need to obtain permission directly from the copyright holder. To view a copy of this licence, visit http://creativecommons.org/licenses/by/4.0/.

\section{References}

1. Bortolini M, Galizia F, Mora C (2018) Reconfigurable manufacturing systems: literature review and research trend. J Manuf Syst 49:93-106. https://doi.org/10.1016/j.jmsy.2018.09.005

2. Bergs T, Hermann L, Rey J, Barth S (2020) Methodology for the identification of alternative manufacturing changes for safetycritical components. Prod Eng Res Devel 14:297-307. https:// doi.org/10.1007/s11740-020-00960-1

3. Hermann L, Weber, A, Beckers A, Barth S, Bergs T (2021) Timeand cost-efficient specification and evaluation of manufacturing changes through iterative information acquisition. In: Procedia CIRP, 54th CIRP conference on manufacturing systems, vol 104, pp 176-181. https://doi.org/10.1016/j.procir.2021.11.030

4. Bergs T, Hermann L, Rey J, Barth S (2020) Identification of unintended effects caused by adaptations of manufacturing process sequences for safety-critical components. Procedia CIRP 93:1019-1024. https://doi.org/10.1016/j.procir.2020.03.013

5. Klocke F, Müller J, Mattfeld P, Kukulies J, Schmitt RH (2018) Integrative technology and inspection planning — a case study in 
medical industry. J Manuf Sci Eng 140:1-11. https://doi.org/10. $1115 / 1.4039114$

6. Hermann L, Rey J, Barth S, Bergs T (2020) Systematic generation of manufacturing changes for safety-critical components. J Manuf Syst 56:270-280. https://doi.org/10.1016/j.jmsy.2020.06.008

7. International Organization for Standardization (2015) Aerospace series - quality systems - first article inspection requirements

8. DIN EN ISO (2016) Medical devices—quality management systems-requirements for regulatory purposes

9. Ehrlenspiel K, Kiewert A, Lindemann U, Mörtl M (2020) Kostengünstig Entwickeln und Konstruieren: Kostenmanagement bei der integrierten Produktentwicklung, 8th edn. Springer, Berlin, Heidelberg

10. Verna E, Genta G, Galetto M, Franceschini F (2021) Inspection planning by defect prediction models and inspection strategy maps. Prod Eng Res Devel 15:897-915. https://doi.org/10.1007/ s11740-021-01067-x

11. Schuh G (2011) Technologiemanagement: Handbuch Produktion und Management 2, 2nd edn. VDI-Buch Springer-Verlag, Berlin, Heidelberg

12. Koch J (2017) Manufacturing change management-a processbased approach for the management of manufacturing changes. Dissertation, Technische Universität München

13. Marks P, Hoang XL, Weyrich M, Fay A (2018) A systematic approach for supporting the adaptation process of discrete manufacturing machines. Res Eng Design 29:621-641. https://doi.org/ 10.1007/s00163-018-0296-5

14. Karl F, Reinhart G (2015) Reconfigurations on manufacturing resources: identification of needs and planning. Prod Eng Res Dev 9:393-404. https://doi.org/10.1007/s11740-015-0607-x

15. Hoang X-L, Fay A, Marks P, Weyrich M (2017) Generation and impact analysis of adaptation options for automated manufacturing machines. In: 22nd IEEE international conference on emerging technologies and factory automation (ETFA), pp 1-8. https:// doi.org/10.1109/ETFA.2017.8247673

16. Masood T, Kern M, John Clarkson P (2021) Characteristics of changeable systems across value chains. Int J Prod Res 59:16261648. https://doi.org/10.1080/00207543.2020.1791997

17. Cichos D, Aurich JC (2017) Integration of a physical model into the realization of engineering changes in manufacturing systems.
AMM 869:159-166. https://doi.org/10.4028/www.scientific.net/ AMM.869.159

18. Plehn C (2018) A method for analyzing the impact of changes and their propagation in manufacturing systems. Dissertation, Technische Universität München

19. Müller J (2018) Integrative Gestaltung von Fertigungsprozessund Prüffolgen für sicherheitskritische Bauteile. Dissertation, RWTH Aachen

20. Schmidt J (2015) Integrierte Arbeitsplanung und Fertigungssteuerung auf Basis von Zustandsinformationen. Dissertation, Leibniz Universität Hannover

21. Denkena B, Dittrich M-A, Jacob S (2019) Methodology for integrative production planning in highly dynamic environments. Prod Eng Res Dev 13:317-324. https://doi.org/10.1007/ s11740-019-00889-0

22. Beckers A, Stauder L, Grünebaum T, Barth S, Bergs T (2021) Design of economically-optimized manufacturing process sequences using cross-process models. CIRP J Manuf Sci Technol 33:15-29. https://doi.org/10.1016/j.cirpj.2021.02.008

23. Basse I, Jansen C, Schmitt S, Schmitt R (2013) A decision model for cost-optimized inspection planning. In: 2013 international conference on engineering, pp 1-15. https://doi.org/10.1109/ITMC. 2013.7352604

24. Wuest T, Irgens C, Thoben K-D (2014) An approach to monitoring quality in manufacturing using supervised machine learning on product state data. J Intell Manuf 25:1167-1180. https://doi.org/ 10.1007/s10845-013-0761-y

25. Hasegawa M, Seireg A, Lindberg RA (1976) Surface roughness model for turning. Tribol Int 9:285-289. https://doi.org/10.1016/ 0301-679X(76)90019-0

26. Singh D, Rao PV (2007) A surface roughness prediction model for hard turning process. Int J Adv Manuf Technol 32:1115-1124. https://doi.org/10.1007/s00170-006-0429-2

Publisher's Note Springer Nature remains neutral with regard to jurisdictional claims in published maps and institutional affiliations. 